\title{
Fuzzy Synthetic Evaluation of Relay Protection based on Variable Weight Value
}

\author{
Renjie Song and Yuming Chen \\ College of Information Engineering, Northeast Dianli University, Jilin 132012, \\ China \\ chenyuming0216@163.com
}

\begin{abstract}
To improve the accuracy of the state evaluation is the most important for the relay protection state maintenance. Therefore, a fuzzy synthetic evaluation of relay protection based on variable weight value is proposed in this paper. Pick up the major factor of the device and secondary circuit of relay protection to establish a reasonable index system of state evaluation. Then according to the variable weight comprehensive concept of factor space theory, we introduce the balance function to adjust the weight coefficient of each factor. Final, get the membership function in triangle trapezoid distribution to show the condition information, and select synthetic operator, then an evaluation system of relay protection with objectively and accurately is proposed. Running examples show that after dealing with the variable weight, some factors weight value become larger or smaller, so running state of evaluate factor may be changed, make it close to the real running state.
\end{abstract}

Keywords: Relay protection; State evaluation; Variable weight Fuzzy comprehensive evaluation

\section{Introduction}

State maintenance is base of state evaluation of protection relays and secondary circuit, also is the important basis of maintenance decision. The results of the state evaluation will effects the consequence and quality of the protection relays and secondary circuit directly, and it is related to the safe and stable operation of power grid. In state evaluation, the relationship of each state as well as the relationship of each state variables and evaluate results should be considered adequately. Protection relays and secondary circuit involved a large amount of state factors, imperceptible changes of every state factors will effect on the results of the state evaluation immediately or indirectly. In the past, constant weight coefficient was used for the fuzzy synthetic evaluation, when individual significance parameters seriously deviated from its normal, constant weight could not reflect the impact of the operation of protection relays objectively. When a minor constant weight state factor was changed, the results of the state evaluation may be in a normal level. Therefore, some wrong maintenance plan will be decided which would be lead to an unnecessary damage.

In this paper, on the basis of improvement the evaluation index system of relay protection status, entropy method is used to calculate the constant weight, the balance function is used for adjust the variable weight coefficients, choosing appropriate synthetic operator. Then a comprehensive status evaluation of protection relays and secondary circuit model is proposed. The condition assessing model could reflect the real running condition of protection relays and secondary circuit. 


\section{The Index System of Relay Protection Status Evaluation}

The factor index which can be effect the protection relays and secondary circuit is an enormous datasets and it is variance between each factors. Hence, choose the major factor of state variables is difficult. In this paper, based on the principle of comprehensiveness, accuracy and operability, combining with historical operation data and real time data to establish the index system of relay protection status evaluation. Especially, the real-time data of temperature can be only reflect the equipment running status, unable reflect the aging degree of the device. When the device temperature is in an abnormal state for a long time, it has a serious harmful effect on the aging degree of the device. As a consequence, the temperature history balance offset is used for the evaluation factor of the historical operation data. As Figure 1 shows that the index system of relay protection status evaluation can satisfy the online status evaluation.

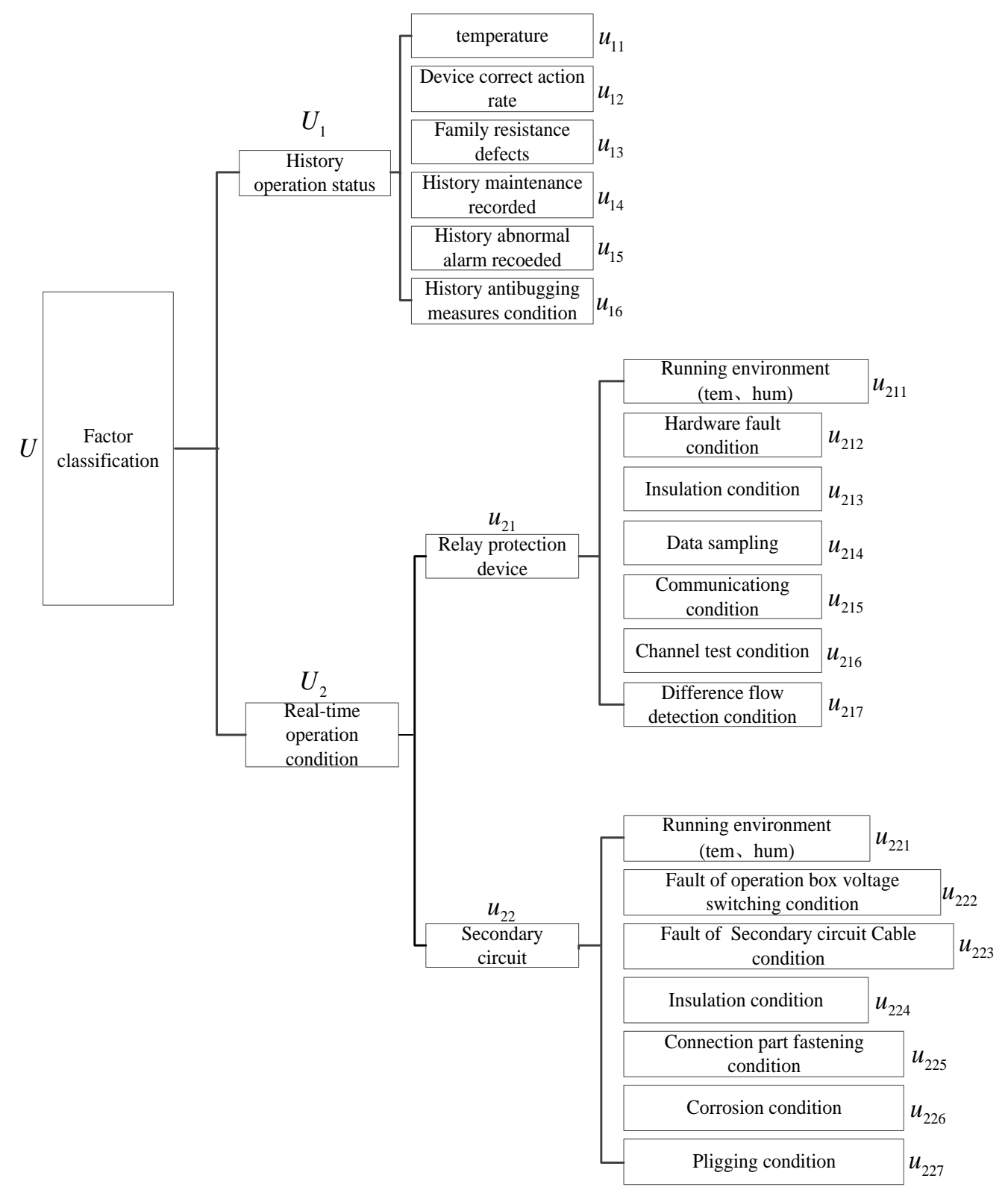

Figure 1. Index System of Relay Protection State Evaluation 


\section{Variable Weight Coefficients of the Comprehensive Evaluation}

\subsection{Constant Weight Coefficient}

In this section, we give a review of entropy method to confirm constant weight. Analytic Hierarchy Process is in common use. Usually, corresponding weights is given by experts. The strong subjective color and the difference brought by every experts experience make the results of evaluation is different. In this paper, using entropy method to confirm constant weight in order to avoid the above disadvantages. The algorithm is described as follows:

(1) Build a data matrix

$$
U=\left(\begin{array}{ccc}
u_{11} & \cdots & u_{1 m} \\
\vdots & \vdots & \vdots \\
u_{n 1} & \cdots & u_{n m}
\end{array}\right)_{n \times m}
$$

Of the above factors, $U_{i j}$ denotes the value of factors $j$ in state variables $i$.

(2) Data normalization treatment

Because each evaluation factors do not have metrizability, we can not directly use the initial factors of each state variables fuzzy comprehensive evaluation. We need to eliminate the dimension, magnitude and the factor type of each state variables and then fuzzy comprehensive evaluation. It is also called standardized processing.

For positive indicators

$$
u_{i j}^{*}=\frac{x_{i j}-\min \left\{x_{j}\right\}}{\max \left\{x_{j}\right\}-\min \left\{x_{j}\right\}}
$$

For negative indicators

$$
u_{i j}^{*}=\frac{\max \left\{x_{j}\right\}-x_{i j}}{\max \left\{x_{j}\right\}-\min \left\{x_{j}\right\}}
$$

Let $U_{i j}$ denote standardized data after standardized processing.

(3) The proportion of state variables $i$ index account for factors $j$ is calculated.

$$
X_{i j}=\frac{u_{i j}^{*}}{\sum_{i=1}^{n} u_{i j}^{*}} \quad(j=1,2, \cdots m)
$$

(4) Information entropy and redundancy of entropy of factors ${ }_{j}$ is calculated.

Information entropy

$$
e_{j}=-k \sum_{i=1}^{m} X_{i j} \times \ln X_{i j}
$$

Redundancy of entropy

$$
d_{j}=1-e_{j}
$$

(5) Constant weight coefficient

$$
w_{i}=\frac{d_{j}}{\sum_{j=1}^{m} d_{j}}, j=1,2 \cdots m
$$

Entropy weight, as an objective weight method, fully consider the information provided by evaluate index, avoid the deviation by artificial factor.

\subsection{Variable Weight Coefficient}

In order to evaluate the real running condition of the protection relays and secondary circuit, the state variables of the protection relays and secondary circuit is divided into several evaluation factors. Comprehensive assess the evaluation factors and then we can obtain the results of evaluation factors. Final, the running state of the protection relays 
and secondary circuit is obtained by fuzzy comprehensive evaluation. On operation processing, although entropy method improve too dependent on subjective factors to some extent, when some small weight value diverge from normal values seriously, assessment index of evaluation factors can not be highlighted through fuzzy operator. However, protection relays and secondary circuit still stay in normal state. Thus, only use constant weight by entropy method cannot reflect the real running condition of the protection relays and secondary circuit, balance function is introduced to adjust weight coefficient.

Variable weight with balance function realized real-time adjustment of weight coefficient. It can reflect the proportionality of each factors in comprehensive evaluation, solve the problem that the difference between evaluation result and running state caused by the change of smaller state evaluation factors. Variable weight formula as follows:

$$
w_{i}^{v}=\frac{w_{i}}{x_{i}} / \sum_{k=1}^{n} \frac{w_{k}}{x_{k}}
$$

Here, $w_{i}^{v}$ is the mean of variable weight coefficient of state variables $i ;{ }_{x_{i}}$ is the mean of rating value of state variables $i ; n$ is the means of number of state variables; $w_{i}$ is the means of constant weight coefficient of state variables $i$.

Balance function $B_{\Pi_{\tau}}$

$$
B_{\Pi_{\mathrm{T}}}=\prod_{i=1}^{m} x_{i}^{T}(T>0)
$$

Introduce balance function ${ }_{B_{\Pi_{\mathrm{T}}}}$ into variable weight formula

$$
w_{i}^{v}=\frac{w_{i}}{x_{i} \sum_{k=1}^{m} w_{k} x_{k}^{-1}}
$$

Variable weight formula after introducing balance function could reflect proportionality of each factor state.

\section{State Evaluation Steps}

\subsection{Factor and Comment Sets}

As Figure 1 shows that the evaluation results $U$ of the protection relays and secondary circuit includes two parts: 1) the history operation condition $_{U_{1}}$;2) the actual operation condition $U_{2}$. History operation condition $U_{1}$ includes device $U_{21}$ and secondary circuit $U_{22}$.Therefore, factor sets denote $U=\left\{U_{1}, U_{2}\right\}$, where $U_{1}=\left\{u_{i}, i=1 \ldots 6\right\}$, $U_{2}=\left\{u_{21}, u_{22}\right\}$ and $u_{21}=\left\{u_{211}, i=1 \ldots .7\right\} u_{22}=\left\{u_{22}, j=1 \ldots .7\right\}$.

Considering that maintenance strategy and maintenance schedule, the running state of the protection relays and secondary circuit is divided into well, normal, similar to normal, attention, similar to attention, abnormal, severely subnormal. Operation and maintenance staffs should be pay attention both similar to normal and similar to attention, which is in

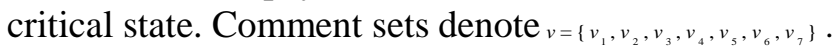

\subsection{Membership Function}

Membership function is the key to use fuzzy geometric theory to solve practical problems. Fuzzy statistical method, citing instances method and expert experience method is common used. In engineering, the distribution form membership function is trapezium distribution, triangle distribution, normal distribution etc. Considering that state distribution of relay protection disobeys an idealized trapezium distribution; Normal distribution shield information result in membership degree is lower; the two ends of triangle distribution have no fuzzy set center. In order to improve the rationality and 
accuracy of condition evaluation in the protection relays, using improved triangle and trapezium distribution could expressed membership degree accurately. The paper select improved triangle and trapezium distribution model, each state level fuzzy set have a center. That is the maximum point of membership degree peak. Figure 2 shows that the triangle and trapezium distribution.

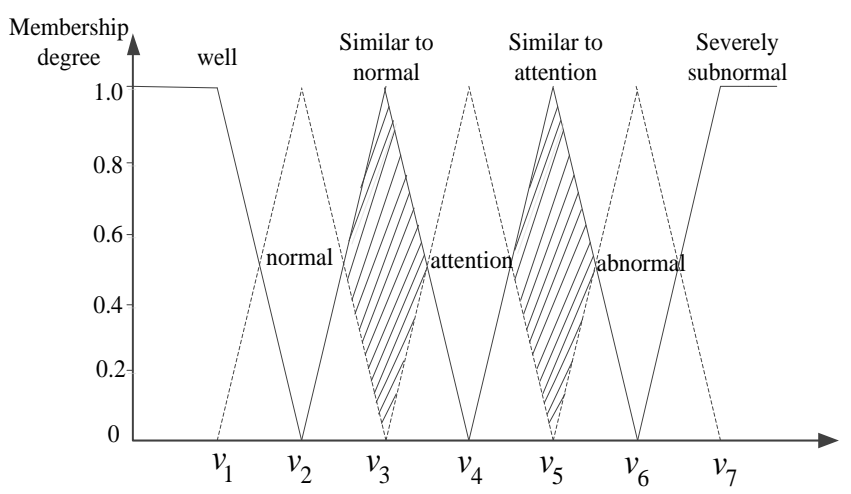

\section{Figure 2. Membership Distribution of Fuzzy Trapezium and Triangle} Distribution

The expression of triangle and trapezium distribution is

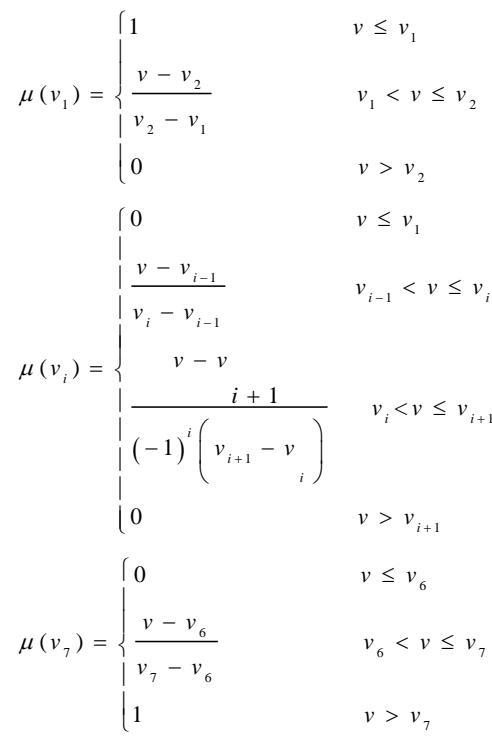

As Fuzzy relation matrix $R_{i}=\left\{r_{i 1}, r_{i 2}, r_{i 3} \ldots r_{i 7}\right\}$, single factor evaluation matrix $R$

$$
R=\underset{\sim}{R=}\left[\begin{array}{cccc}
r_{11} & r_{12} & \cdots & r_{17} \\
r_{21} & r_{22} & \cdots & r_{27} \\
\vdots & \vdots & & \vdots \\
r_{m 1} & r_{m 2} & \cdots & r_{m 7}
\end{array}\right]
$$

Fuzzy comprehensive evaluation of the weight sets ${ }_{A}$ confirmed by variable weight coefficient formula and single factor evaluation matrix $R$, then the result could be obtained the evaluation fuzzy subsets $\underline{B}$.

\subsection{Results of Synthesis Fuzzy Comprehensive Evaluation}

This paper use the model $\mathrm{M}(\cdot, v)$ to synthesis, the data in this operator model directly decided by matrix $\mathbb{R}_{\sim}$ in $b_{j}$ may not be the maximum factors in each column. Not only 
requires $r_{i j}$ large, and requires $a_{i}$ should be large. Put the $r_{i j}$ revised to $r_{i j}{ }^{a_{i}}$, because $r_{i j}$ had restricted functionality, single factor can better reflect the importance of the evaluation results. Highlights the information in the secondary factors to judge, calculation formula of the $\operatorname{model}_{b}$

$$
b_{j}=\hat{i=1}_{i=1}^{n}\left(r_{i j}^{a_{i}}\right), \quad j=1,2, \ldots, m
$$

According to comprehensive evaluation of the synthetic operator $\mathrm{M}(\cdot, v)$ in the protection relays and secondary circuit, we can get a scientific and rational conclusion. The result can be very intuitive to reflect the real running condition of the protection relays and secondary circuit.

\section{Case Analyses}

Using index system and fuzzy synthetic evaluation method based on variable weight value above mention in this paper, to evaluate a relaying protection device of a substation as an example to comprehensive analysis running condition.

\subsection{Fuzzy Relationship Matrix}

According to monitoring of the actual data of the relay protection device evaluation state variable, substitution membership function of each state of formula (11) to obtain membership degree of each factor. In table 1 , fuzzy relation matrix ${ }_{R}$ of running state is obtained.

Table 1. Evaluation State Variables of Protection Relays

\begin{tabular}{|c|c|c|c|c|c|c|c|c|c|}
\hline \multirow{2}{*}{ State variables } & \multirow{2}{*}{ Evaluation Content } & \multirow{2}{*}{$\begin{array}{l}\text { Factor } \\
\text { set }\end{array}$} & \multicolumn{7}{|c|}{ Membership degree } \\
\hline & & & $v_{1}$ & $v_{2}$ & $v_{3}$ & $v_{4}$ & $v_{5}$ & $v_{6}$ & $v_{7}$ \\
\hline $\begin{array}{l}\text { Device operating } \\
\text { environment }\end{array}$ & $\begin{array}{l}\text { Running environment } \\
\text { (tem, hum) }\end{array}$ & $u_{211}$ & 0.7 & 0.1 & 0.2 & 0 & 0 & 0 & 0 \\
\hline $\begin{array}{l}\text { Hardware fault } \\
\text { condition }\end{array}$ & $\begin{array}{l}\text { The case of software and } \\
\text { hardware equipment failure }\end{array}$ & $u_{212}$ & 0.5 & 0.3 & 0.1 & 0.1 & 0 & 0 & 0 \\
\hline Insulation condition & $\begin{array}{l}\text { Including data in the screen } \\
\text { wiring insulation } \\
\text { protection cabinet and each } \\
\text { plug }\end{array}$ & $u_{213}$ & 0.6 & 0.1 & 0.1 & 0.2 & 0 & 0 & 0 \\
\hline Data sampling & $\begin{array}{l}\text { Sampling analog and } \\
\text { digital sampling }\end{array}$ & $u_{214}$ & 0.5 & 0.2 & 0.1 & 0.2 & 0 & 0 & 0 \\
\hline $\begin{array}{l}\text { Communication } \\
\text { condition }\end{array}$ & $\begin{array}{l}\text { Communication condition } \\
\text { between monitoring } \\
\text { background and protection } \\
\text { information }\end{array}$ & $u_{215}$ & 0.6 & 0.2 & 0.1 & 0.1 & 0 & 0 & 0 \\
\hline $\begin{array}{l}\text { Channel test } \\
\text { condition }\end{array}$ & $\begin{array}{l}\text { HF channel test and Fiber } \\
\text { channel test }\end{array}$ & $u_{216}$ & 0.7 & 0.1 & 0.1 & 0.1 & 0 & 0 & 0 \\
\hline $\begin{array}{l}\text { Difference flow } \\
\text { detection condition }\end{array}$ & $\begin{array}{l}\text { Main variation flow, bus } \\
\text { difference flow and fiber } \\
\text { longitudinal differential } \\
\text { data }\end{array}$ & $u_{217}$ & 0.4 & 0.4 & 0.1 & 0.1 & 0 & 0 & 0 \\
\hline
\end{tabular}


Table 1 can obtain the fuzzy relation matrix $R_{x}$

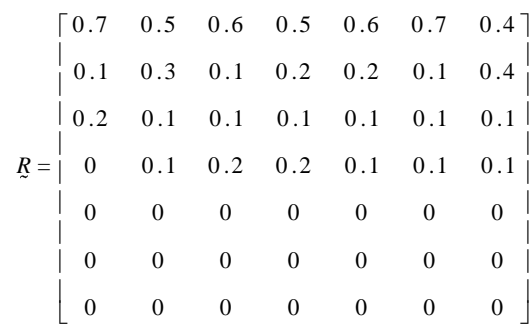

\subsection{Constant and Variable Weight Coefficient}

First, using entropy value method of formula (7) can obtain constant weight coefficient ${ }_{A_{1}}$, using formula (10) can adjust variable weight coefficient ${ }_{A_{2}}$, which is shown in table 2.

Table 2. Constant Weight and Variable Weight Coefficient

\begin{tabular}{|c|c|c|c|c|c|c|c|}
\hline$\Phi \alpha \chi \tau о \rho \sigma \varepsilon \tau$ & $u_{211}$ & $u_{212}$ & $u_{213}$ & $u_{214}$ & $u_{215}$ & $u_{216}$ & $u_{217}$ \\
\hline $\mathrm{Xov} \sigma \tau \alpha \nu \tau$ & 0.315 & 0.39 & 0.15 & 0.09 & 0.013 & 0.032 & 0.01 \\
\hline$\varpi \alpha \rho \iota \alpha \beta \lambda \varepsilon$ & 0.318 & 0.221 & 0.21 & 0.068 & 0.083 & 0.042 & 0.058 \\
\hline
\end{tabular}

Table 2 is express that

Constant weight sets:

$$
A_{1}=\left[\begin{array}{lllllll}
0.315 & 0.39 & 0.15 & 0.09 & 0.013 & 0.032 & 0.01
\end{array}\right]^{\mathrm{T}} ;
$$

Variable weight sets:

$$
\underset{\sim 2}{A}=\left[\begin{array}{lllllll}
0.318 & 0.221 & 0.21 & 0.068 & 0.083 & 0.042 & 0.058
\end{array}\right]^{\mathrm{T}}
$$

\subsection{Fuzzy Comprehensive Evaluation}

According to formula (13), comprehensive evaluation result of relay protection device in constant weight coefficient and variable weight coefficient obtained respectively, result are as follow.

$$
\begin{aligned}
b_{1} & =\underset{\sim}{R} \circ{ }_{\sim 1}^{A}=\left[\begin{array}{lllllll}
0.4966 & 0.2635 & 0.1419 & 0.088 & 0 & 0 & 0
\end{array}\right]^{\mathrm{T}} \\
b_{2} & =\underset{\sim}{R}{ }^{\circ} \underset{\sim}{A}=\left[\begin{array}{lllllll}
0.5679 & 0.1667 & 0.1667 & 0.1056 & 0 & 0 & 0
\end{array}\right]^{\mathrm{T}}
\end{aligned}
$$

Membership degree of protection relay devices running state by the constant weight coefficient belong to well condition is 49.66 percent, membership degree is 56.79 percent by the variable weight coefficient. The membership degree of protection relay devices belong to well condition is maximum, the weight coefficient is similar to running state before adjust the weight coefficient. It is explanation that variable weight process could be made sure the accuracy of the evaluation results. The membership degree in the normal state is falling after variable weight process, the condition of similar to normal and attention membership degree is increase. The results show that, although the overall running state is in well condition, some factors index of state variable is falling. Due to the constant weight coefficient is small, the relay protection devices may be in well condition. The variable weight increased its weight coefficient and the relay protection may be in poor condition, close to the real running state, convenient for the efficiency of maintenance work.

\section{Conclusions}

(1) Establish the evaluation index system by extracting important factors from many important influence factors in the relay protection device and secondary circuit. The index 
system according to the text is combined with the running status of the history and the actual operation situation. It will be a great help for the accurate judgment of the actual operation of the relay protection device to refer to the running status of the history when monitoring the actual operation situation.

(2) Put forward the weight coefficient in the way that use the balance function to construct the adjustment factors of variable weight based on constant weight. Balance function, one of the important modeling principle of variable weight synthesis theory, shows the balance among all the elements in the comprehensive decision, improves the problem that when the constant weight coefficient contributes to the performance decline of some aspects of the relay protection device, the overall evaluation may still at the normal level, and improves the accuracy of the judgment results.

(3) This paper uses the triangular fuzzy trapezoidal membership function to construct the fuzzy evaluation matrix of state variables. Considering that the state distribution of relay protection will not obey the ideal trapezoidal distribution when operating really, we combine the characteristics of the triangle distribution and trapezoid distribution, and use the improved triangular trapezoidal distribution to express the state of the distribution and gradation more accurately

(4) By using $\mathrm{M}(\cdot, v)$ to compose the operation can reflect the importance of the evaluation results of single factors in the fuzzy comprehensive operation.

This paper presents a fuzzy method to evaluate the state of relay protection based on variable weight coefficient. In some degree, it can improve the accuracy of state evaluation of the relay protection device and secondary circuit and provide reliable reference basis for the maintenance of relay protection condition.

\section{References}

[1] X. X. Fu, C. X. Tian and Z. C. Sheng, "Overview of research on state evaluation of relaying protection system", Power System Protection and Control, vol. 42, no, 5, (2014), pp. 51-58.

[2] L. Li, Z. Deng and X. Longjun, "A Condition Assessment Method of Power Transformers Based on Association Rules and variable Weight Coefficients", Proceedings of the CSEE, vol. 33, no, 24, (2013), pp. 152-159.

[3] W. Jiang, C. Z. Xiang and H. C. Chao, "Status evaluation of protective relays based on the membership function in fuzzy normal distribution”, Power System Protection and Control, vol. 40, no. 5, (2012), pp. 48-52.

[4] L. Lei, X. X. Fu and S. Z. Jianm "Fuzzy Synthetic Evaluation of Transformer Condition with Variable Weight Value", High Voltage Apparatus, vol. 50, no. 7, (2014), pp. 100-105.

[5] P. Z. Feng and Y. M. Yu, "Status Evaluation Method of Relay Protection Based on the Variable Weight Theory", ELECTRIC POWER, vol. 50, no. 7, (2014), pp. 100-105.

[6] Z. J. Chun, Q. H. Ping and Q, J. Chuan, "The Construction of Balance Function in Variable Weight Evaluation", Fire Control and Command Control, vol. 32, no, 7, (2007), pp. 107-110.

[7] K. Jiang, Chanan, Singh Fellow, IEEE. "New Models and Concepts for Power System Reliability Evaluation Including Protection System Failures", IEEE Transactions on Power Systems, vol. 26, no. 4, (2011), pp. 1845-1854.

[8] L. Wenqi, "Balanced Function and Its Application for Variable Weight Synthesizing", System Engineering Theory and Practice vol. 17, no. 4, (1997), pp. 58-64.

[9] L. Y. Xin, S. Feng and J. Shuai, "A fuzzy evaluation algorithm for condition monitoring of smart substation relay protection", Power System Protection and Control, vol. 42, no. 3, (2014), pp. 37-41.

[10] L. W. Chao, L. F. Peng and Z. X. Feng, "Application of Fuzzy Clustering Method in the Conditionbased Maintenance ( CBM ) of Relay Protection", Proceedings of the CSU-EPSA, vol. 25, no. 2, (2013), pp. 93-97. 


\section{Authors}

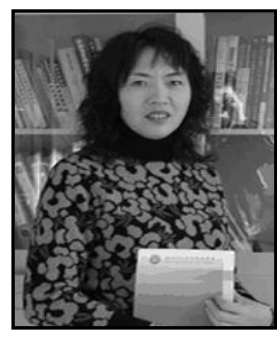

Renjie Song, (1963), female, master, professor, Research direction:

Computer applications in power system, Email:srj1963331@sina.com.

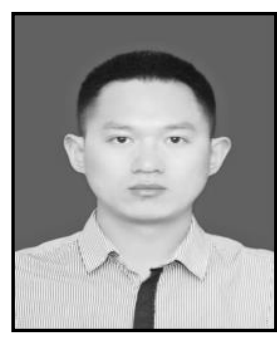

Yuming Chen, (1990), male, master degree candidate, Research direction: Artificial intelligence application in power system, Email:chenyuming0216@163.com. 
International Journal of Multimedia and Ubiquitous Engineering

Vol.10, No.11 (2015) 